\title{
Knowledge, Attitudes and Practices of COVID-19 Among Urban and Rural Residents in China: A Cross-sectional Study
}

\author{
Shaoting Yue ${ }^{1} \cdot$ Jun Zhang ${ }^{1}$ (1) $\cdot$ Mi Cao ${ }^{1} \cdot$ Bowen $^{\text {Chen }}{ }^{2}$ \\ Published online: 5 August 2020 \\ ○) Springer Science+Business Media, LLC, part of Springer Nature 2020
}

\begin{abstract}
This study aims to understand the knowledge, attitudes and practices of COVID-19 in the Chinese context and to provide insights for developing targeted outbreak prevention and control measures among the general public. We conducted an online survey of urban and rural residents in Henan Province. A total of 517 valid questionnaires were collected via the online platform. The mean scores for knowledge and practice were 5.57/9 and 2.04/3, respectively. More than $90 \%$ of the participants believed COVID-19 was serious and preventable, were concerned about the disease process, and actively engaged in learning related knowledge. Our results showed that the COVID-19 knowledge level was significantly different among groups with different ages, genders, education levels and marital statuses; COVID-19 practice was significantly different among different regions. Multiple linear regression analysis showed that education level, female sex, unmarried status, and health care worker status had a significant impact on COVID-19 knowledge; urban area was associated with a higher practice score; COVID19 knowledge was significantly associated with residents' attitude toward preventive measures that can prevent COVID-19 infection; urban area was significantly related to the willingness to go to a fever clinic to check for suspected infection. We found that Chinese urban and rural residents held a moderate level of COVID-19 knowledge and practice and showed a positive attitude toward the disease. It is necessary to develop relevant education programs targeting the general population in China to improve COVID-19-related knowledge, attitudes and practices, particularly for rural and undereducated residents.
\end{abstract}

Keywords COVID-19 $\cdot$ Residents $\cdot$ Knowledge $\cdot$ Attitude $\cdot$ Practice

\section{Introduction}

At the end of Dec 2019, Wuhan, as a central part of China, had the first outbreak of coronavirus disease (COVID-19) [1]. The disease then quickly spread to other parts of China. As an adjoining province, Henan had 206 confirmed cases

Jun Zhang

catherinezhang2010@126.com

Shaoting Yue

1245664908@qq.com

$\mathrm{Mi} \mathrm{CaO}$

1826588854@qq.com

Bowen Chen

823996171@qq.com

1 Faculty of Nursing, Wuhan University School of Health Sciences, 115 Donghu Rd, Wuchang District, Wuhan 430071, China

2 School of Public Health, Sun Yat-sen University, 74 Zhongshan 2nd Rd, Guangzhou 510080, China on January 29, 2020, and 1272 confirmed cases on February 29 [2]. COVID-19 is a public health emergency of international concern. On 2 May 2020, there were 3,272,202 confirmed cases of COVID-19 and 230,104 deaths [3]. COVID19 was classified as infectious disease class B; however, due to the high contagious risk, the prevention and control management level was designated level A [4].

Under such a severe situation, little is known about the status regarding knowledge, attitude and practice (KAP) toward COVID-19 among residents from different regions and countries. The purpose of the study is to explore the level of knowledge, attitude and practice related to COVID19 among the general population in China to provide a scientific basis for preventing and controlling this major epidemic in China and other parts of the world. 


\section{Methods}

\section{Study Design and Population}

This cross-sectional study was conducted from February 16 to 18 in Henan Province, China's most populous province, adjacent to the city of Wuhan. Convenience and snowball sampling were used. People aged 15 years or older with access to the computer and/or mobile phone were invited to participate in the study. The sample size was calculated as 5-10 times the number of questionnaire items, which was 360 . Considering the possibility of $20 \%$ invalid questionnaires, we included 517 in the final sample.

\section{Measurement Tool}

We designed an online questionnaire by referring to the health education manual of coronavirus disease published by the China Health Education Center [5]. The online questionnaire was produced through the Wenjuanxin electronic platform (www.wjx.cn). The questionnaire consisted of 2 sections with a total of 28 items. The first part was about the source of COVID-19 knowledge and demographic information, such as age, gender, educational level, marital status, occupation and area of residence (urban or rural). The second part was KAP with a total of 20 items. The knowledge dimension included 9 items, the attitude dimension covered 8 items, and the practice dimension covered 3 items. A correct answer was assigned 1 point, and an incorrect answer was given 0 points.

\section{Data Collection}

Due to the epidemic and home quarantine, participants could not be reached face to face. We collected data by sharing the online questionnaire on several social networking platforms (e.g., WeChat, QQ). On the first page of the questionnaire, the research purpose and research procedure were provided.

\section{Statistical Analysis}

Both descriptive analysis and inferential analysis were used. Frequencies $(\mathrm{N})$, percentages (\%), mean value (M) and standard deviation (SD) were used to present demographic characteristics and address the results regarding the level and distribution of knowledge, attitude and practice toward COVID-19. The nonparametric test was used to explore the association between demographic characteristics variables and COVID-19 knowledge and practice scores. Multiple linear regression analysis was used to identify the factors associated with knowledge and practice of COVID-19.
Binary logistic regression was used to identify influencing factors of COVID-19 attitude. All data were analyzed using SPSS 20.0 , with $\mathrm{P}<0.05$ considered statistically significant (two-sided).

\section{Ethical Consideration}

Permission to conduct the study was sought from the Wuhan University Medical School Ethics Committee. A statement of informed consent was provided on the first page of the questionnaire. Informed consent was obtained automatically once the adult survey participants understood the content and volunteered to fill in the online survey. For participants aged $15-18$, the researchers would specifically contact the guardian of minors with WeChat or QQ to obtain their informed consent for their children's participation in the study. Once they agreed and the minors submitted the questionnaire, informed consent was obtained. Maximum efforts were used to protect participants' anonymity and confidentiality. All data were stored in a password-locked computer and only available to the research team. The data were de-identified, and only aggregate data were used for the presentation of the results.

\section{Results}

\section{Descriptive Characteristic Results}

A total of 564 questionnaires were distributed via the internet, and 517 questionnaires were returned with a response rate of $92 \%$. The 517 participants were from 17 different cities in Henan Province, with 158 (31\%) urban residents and $359(69 \%)$ rural residents. In total, 278 (54\%) men and $239(46 \%)$ women finished the survey. The distribution of age, gender, region, occupation, education level and marital status are presented in Table 1.

\section{The Correct Rate of COVID-19 Knowledge, Attitude and Practice}

The scores of knowledge and practice were $5.57 \pm 1.53$ and $2.04 \pm 0.81$, respectively, suggesting that the overall correct rates of knowledge and practice were $61.9 \%$ and $68.0 \%$, respectively. Regarding knowledge, almost all participants (95.3\%) stated that they knew how to properly wear disposable surgical masks/surgical masks. In terms of the causes, clinical symptoms, incubation period, transmission route, treatment of COVID-19, and proper diet, the correct rate was $23.4 \% \sim 84.3 \%$. Among them, "The transmission route of COVID-19" had the lowest correct rate, followed by "Clinical symptoms". "How long is the incubation period of COVID-19" had the highest correct rate. 
Table 1 Demographic characteristics of the participants $(n=517)$

\begin{tabular}{|c|c|}
\hline Demographic characteristics & $\begin{array}{l}\text { Number of } \\
\text { participants } \\
(\%)\end{array}$ \\
\hline \multicolumn{2}{|l|}{ Age (years) } \\
\hline $15-20$ & $73(14.12 \%)$ \\
\hline $21-30$ & $272(52.61 \%)$ \\
\hline $31-40$ & $5(9.86 \%)$ \\
\hline $41-50$ & $83(16.05 \%)$ \\
\hline $51-60$ & $38(7.35 \%)$ \\
\hline \multicolumn{2}{|l|}{ Gender } \\
\hline Female & $278(53.77 \%)$ \\
\hline Male & $239(46.23 \%)$ \\
\hline \multicolumn{2}{|l|}{ Area of residence } \\
\hline Urban & $158(30.56 \%)$ \\
\hline Rural & $359(69.44 \%)$ \\
\hline \multicolumn{2}{|l|}{ Occupation } \\
\hline Student & $188(36.40 \%)$ \\
\hline Farmer & $94(18.20 \%)$ \\
\hline Worker & $49(9.50 \%)$ \\
\hline Teacher & $24(4.64 \%)$ \\
\hline Health care worker & $33(6.38 \%)$ \\
\hline Business/service personnel & $52(10.06 \%)$ \\
\hline Professional and technical personnel & $19(3.68 \%)$ \\
\hline Civil servants and related personnel & $5(0.97 \%)$ \\
\hline Government official & $3(0.58 \%)$ \\
\hline Production and transportation Equipment personnel & $2(0.39 \%)$ \\
\hline Retiree & $2(0.39 \%)$ \\
\hline Else & $47(9.09 \%)$ \\
\hline \multicolumn{2}{|l|}{ Education level } \\
\hline Primary school and below & $37(7.16 \%)$ \\
\hline Middle school & $98(18.96 \%)$ \\
\hline Senior high school/vocational school & $132(25.53 \%)$ \\
\hline Junior college/bachelor's degree & $197(38.10 \%)$ \\
\hline Master's degree and above & $53(10.25 \%)$ \\
\hline \multicolumn{2}{|l|}{ Marital status } \\
\hline Never married & $302(58.41 \%)$ \\
\hline Married & $204(39.46 \%)$ \\
\hline Divorced & $7(1.35 \%)$ \\
\hline Widowed & $4(0.77 \%)$ \\
\hline
\end{tabular}

For attitude, all the respondents recognized the seriousness of the outbreak. Almost all (99.6\%) of the residents believed that preventive measures, such as going outdoors less, washing hands frequently and wearing masks could prevent the infection of COVID-19. They claimed that the prevention and control measures taken by the government had raised their awareness of the disease. Almost all (99.6\%) would choose to immediately report or cut off contact with people around them returning from Wuhan or its surrounding areas; $99.4 \%$ were concerned about the deterioration of the epidemic; $95.7 \%$ were worried that they themselves or family members would be infected; and $92.6 \%$ of the residents would actively acquire relevant knowledge. However, there was also a high percentage of participants (43.7\%) who would not want to go to the fever clinic to check for suspected infection.

Regarding practice, $87.8 \%$ of the respondents bought N95 masks/disposable surgical masks/surgical masks, $45.1 \%$ washed their hands using a 7-step protocol, and 70.4\% covered their nose and mouth with either paper tissues or elbows while coughing or sneezing (Table 2).

\section{The Influencing Factors of COVID-19 Knowledge, Attitude and Practice}

The results of univariate analysis showed that COVID19 knowledge was significantly different among different groups by age, gender, education level, and marital status $(\mathrm{P}<0.05)$. The COVID-19-related practices were significantly different among different areas $(\mathrm{P}<0.05)$.

The multiple linear regression analysis showed that respondents with the male gender (vs. female, $\beta$ : -0.305 , $\mathrm{P}<0.05$ ) and the marital status of married (vs. unmarried, $\beta:-0.421, \mathrm{P}<0.05)$ presented lower knowledge scores. The occupation of health care workers (vs. farmers, $\beta$ : 0.924 , $\mathrm{P}<0.05)$ and higher education levels indicated higher knowledge (Table 3).

Logistic regression analysis found that the COVID-19 knowledge score (OR 0.571, $\mathrm{P}<0.05)$ was significantly associated with the residents' understanding that preventive measures, such as reducing going out and wearing a mask, can prevent COVID-19 infection; urban area (vs. rural, OR $0.586, \mathrm{P}<0.05)$ was significantly related to the attitude of willingness to go to a fever clinic to check for suspected infection (Table 4). Furthermore, multiple linear regression analysis showed that living in an urban area (vs. rural, $\beta$ : $0.183, \mathrm{P}<0.05)$ was significantly linked to higher practice scores (Table 5).

The main ways Chinese residents knew about COVID-19 were social media (92.0\%), TV (66.2\%), and chatting with others $(53.0 \%)$. Other channels included village radio and websites $(5.8 \%)$.

\section{Discussion}

\section{Current Knowledge, Attitude and Practice Among Chinese Residents}

The scores of knowledge and practice were $5.57 \pm 1.53$ and $2.04 \pm 0.81$, respectively, and the overall correct rates of knowledge and practice were $61.9 \%$ and $68.0 \%$, respectively, suggesting that both the knowledge and practice levels of 
Table 2 Knowledge, attitude and practice of COVID-19 among the participants $(\mathrm{n}=517)$
Items

Correct answer rate/choose "yes" $(\%)$

\begin{tabular}{lr}
\hline Knowledge & \\
Name of COVID-19 & 49.00 \\
The cause of COVID-19 & 49.10 \\
How to properly wear a surgical mask/disposable surgical mask & 95.30 \\
The incubation period of COVID-19 & 84.30 \\
Clinical symptoms of COVID-19 & 41.90 \\
Is there any specific drug to treat COVID-19 & 74.60 \\
The transmission route of COVID-19 & 23.40 \\
Masks that can be used to prevent COVID-19 & 52.80 \\
Proper diet & 42.10 \\
Attitude & \\
Actively acquire COVID-19-related knowledge & 92.60 \\
Worried about infection & 95.70 \\
Concerned about the deterioration of the outbreak & 99.40 \\
Considered the outbreak to be very serious & 100.00 \\
Knew that preventive measures, such as reducing going out and washing hands & 99.60 \\
frequently, can prevent it & \\
Went to the hospital after suspecting infection & 66.30 \\
Immediately report or cut off contact with people returning from Wuhan or its sur- & 99.60 \\
rounding areas & \\
Practice & \\
Purchased a medical surgical mask/disposable surgical mask & 87.60 \\
Covered nose and mouth with tissues or arms while coughing or sneezing & 70.40 \\
Washed hands using 7-step protocol & 45.10
\end{tabular}

\begin{tabular}{|c|c|c|c|c|c|}
\hline Variable & $\begin{array}{l}\text { Partial regres- } \\
\text { sion coefficients }\end{array}$ & Standard error & $\begin{array}{l}\text { Standardized } \\
\text { regression coef- } \\
\text { ficient }\end{array}$ & $\mathrm{T}$ & $P$ \\
\hline Constant & 5.402 & 0.139 & - & 38.826 & 0.000 \\
\hline Gender & -0.273 & 0.125 & -0.089 & -2.174 & $0.030^{*}$ \\
\hline $\begin{array}{l}\text { Education level (Junior } \\
\text { college/bachelor's degree } \\
\text { vs. Primary school and } \\
\text { below) }\end{array}$ & 0.724 & 0.148 & 0.230 & 4.901 & $0.000^{*}$ \\
\hline $\begin{array}{l}\text { Education level (Master's } \\
\text { degree and above vs. Pri- } \\
\text { mary school and below) }\end{array}$ & 1.211 & 0.224 & 0.241 & 5.406 & $0.000^{*}$ \\
\hline Marital status & -0.421 & 0.141 & -0.135 & -2.991 & $0.003^{*}$ \\
\hline Occupation & 0.904 & 0.258 & 0.145 & 3.501 & $0.001 *$ \\
\hline
\end{tabular}

$* P<0.05 ; \mathrm{F}=4.725, \mathrm{P}=0.030, \mathrm{R}^{2}=0.188$, adjusted $\mathrm{R}^{2}=0.180$; adjusted $\mathrm{R}^{2}$ indicated that the equation had a predicted accuracy of $18.0 \%$
Table 3 Multiple linear regression analysis of COVID19 knowledge and related factors urban and rural residents in China were at the medium level. Roy et al. conducted a cross-sectional study in India and showed similar results [6]. Zhong et al. conducted a survey in China showing that the overall correct rate of COVID19 knowledge was $90 \%$, which was much higher than that of our study [7]. The difference might be due to the highly educated sample in Zhong's study. Our study suggested that both urban and rural residents had a lower awareness of the COVID-19 transmission route and clinical symptoms than that reported by Abdelhafiz et al. [8]. China is the first country experiencing the outbreak of COVID-19, and at the beginning, Chinese residents had low experience and awareness about the disease. As an increasing number of countries experience the COVID-19 outbreak and with the 
Table 4 Factors significantly associated with attitudes toward COVID-19

\begin{tabular}{lll}
\hline Variable & OR & $P$ \\
\hline $\begin{array}{l}\text { Preventive measures, such as reducing going out } \\
\text { and washing hands frequently, can prevent it }\end{array}$ & & \\
$\begin{array}{l}\text { COVID-19 knowledge score } \\
\text { Went to the hospital after suspecting infection }\end{array}$ & 0.571 & $0.041^{*}$ \\
Area (urban vs. rural) & 0.586 & $0.018^{*}$ \\
\hline & $*<0.05$; Note. COVID-19 attitude as dependent variable (binary)
\end{tabular}

efforts made by the WHO and local government, COVID19-related knowledge is growing.

Our study found that all the respondents had recognized the seriousness of the disease and were concerned about the deterioration of the epidemic. The great majority of the residents believed that preventive measures could prevent the infection of COVID-19 and thought that people should immediately report or cut off contact with people around them returning from Wuhan or its surrounding areas. These results regarding COVID-19 attitude were similar to those found in a previous study conducted in China [7]. Our study found that a great percentage of people $(43.7 \%)$ would not go to the fever clinic if they had been to Wuhan and had suspected infection. This might be due to a number of reasons, such as contagious disease stigma, fear of social isolation and hospital infection.

In addition, $92.6 \%$ of the residents mentioned that they would actively acquire relevant knowledge. Our findings showed that social media platforms represented the most important source of information (92.0\%), followed by TV (66.2\%). This was consistent with the studies conducted by Abdelhafiz and Ranjan [8,9]. This suggested that social media platforms and TV play important roles in spreading knowledge. Therefore, attention should be paid to strengthening public awareness and health education through multiple channels, especially social media platforms and TV.

As shown in this investigation, after the outbreak, 29.6\% of the respondents did not cover their nose and mouth when they coughed or sneezed, only $45.1 \%$ washed their hands correctly, and $12.4 \%$ of the residents did not wear masks because they had no access to masks. A previous study found that nearly one-fourth of the Bangladeshi residents did not wear masks, and nearly two-thirds of the people sneezed without using their elbows in this pandemic. These dangerous practices were related to low education, marital status, and risk-taking among men and late adolescents [10]. Clearly, the self-protection consciousness of residents still needs to be strengthened, and the protective measures taken by residents still need to be corrected. Studies have shown that health education interventions can improve the knowledge of infectious diseases and compliance with healthy living habits among urban and rural residents [11].

\section{The Factors Influencing COVID-19 Knowledge, Attitude and Practice}

According to our results, age, gender, education, and marital status were influencing factors of COVID-19 knowledge. Unmarried residents had a higher level of knowledge than married residents, which was different from the study conducted in the US [12]. This might be because in our study, $97.5 \%$ of the unmarried residents had a high school diploma or above. In addition, the education level, female sex, and occupation of health care workers had the most significant influences on the knowledge score. The higher the education level was, the higher the knowledge level of COVID-19, which was consistent the finding of a previous study [8]. The knowledge level of medical personnel was higher than that of other professions, and these findings were similar to those of other studies [13]. Therefore, attention should be paid to the health education of the general public, especially those with low educational backgrounds [8]. Women's knowledge level was higher than men's, which was similar to the survey reported by Haque et al. [10]. This may be because $57.2 \%$ of the women in the survey had a bachelor's degree or above. A previous study also showed that women had a higher literacy level of prevention and control of infectious diseases than men, and men were more likely to engage in risky behavior $[10,14]$.

Our survey results showed that urban and rural residents had a positive attitude toward COVID-19. It should be noted that the COVID-19 knowledge score was associated with the optimistic attitude to take protective measures, and people living in urban areas were more likely to seek medical advice than people in rural areas if they suspected infection. This precisely illustrated the significance of improving
Table 5 COVID-19 practice and related factors

\begin{tabular}{llllll}
\hline Variable & $\begin{array}{l}\text { Partial regression } \\
\text { coefficients }\end{array}$ & Standard error & $\begin{array}{l}\text { Standardized regres- } \\
\text { sion coefficient }\end{array}$ & $\mathrm{T}$ & $P$ \\
\hline $\begin{array}{l}\text { Constant } \\
\text { Area (urban vs. } \\
\text { rural) }\end{array}$ & 1.980 & 0.183 & - & 46.349 & 0.000 \\
$\begin{array}{l}* P<0.05 ; \mathrm{F}=5.646, \mathrm{P}=0.018, \mathrm{R}^{2}=0.021, \text { adjusted } \mathrm{R}^{2}=0.017 \text {; adjusted } \mathrm{R}^{2} \text { indicated that the equation had } \\
\text { a predicted accuracy of } 1.7 \%\end{array}$ & 0.077 & 0.104 & 2.376 & $0.018^{*}$ \\
\end{tabular}


COVID-19 knowledge and awareness of the epidemic among residents, especially people living in rural areas $[15,16]$.

Multiple linear regression analysis demonstrated that urban residents have better preventive practices, which may be associated with the fact that urban residents have a high level of health literacy and are exposed to a high degree of health publicity [17]. Rural residents usually have a low level of education, health services are lagging behind, and health publicity is rarely carried out, so it is necessary to improve the prevention and control efforts of rural areas [8].

\section{Conclusion}

Above all, the attitude of urban and rural residents is positive, but the health knowledge level and personal protective behavior need to be improved and strengthened. It is suggested to continue to strengthen the propaganda of relevant knowledge and protective measures through social media, $\mathrm{TV}$ and other channels to promote health education, as well as establish intervention programs targeting the unhealthy behaviors of urban and rural residents.

\section{The Study Limitations}

There were some limitations in this study. All participants came from Henan Province, and most were school students. The sample size was relatively small, the proportion of each age group was uneven, and there was a lack of information about the elderly. We excluded the residents who were unable to participate in the online survey.

Funding This research did not receive any specific grant from funding agencies in the public, commercial, or not-for-profit sectors.

\section{Compliance with Ethical Standards}

Conflict of interest The authors declare that they have no conflicts of interest.

\section{References}

1. Chen, N., Zhou, M., Dong, X., et al. (2020). Epidemiological and clinical characteristics of 99 cases of 2019 novel coronavirus pneumonia in Wuhan, China: A descriptive study. The Lancet (London, England), 395(10223), 507-513. https://doi. org/10.1016/S0140-6736(20)30211-7.

2. Update on covid-19 outbreak in Henan province as of 24:00 on 29 February. (2020). Retrieved May 10, 2020, from https://wsjkw .henan.gov.cn/2020/03-01/1315208.html.

3. Coronavirus disease (COVID-19) Situation Report-103. (2020). Retrieved May 10, 2020, from https://www.who.int/emergencies/ diseases/novel-coronavirus-2019/situation-reports.
4. Announcement by the national health commission. (2020). Retrieved May 10, 2020, from https://www.nhc.gov.cn/jkj/s7916 /202001/44a3b8245e8049d2837a4f27529cd386.shtml.

5. Health education manual of coronavirus disease. (2020). Retrieved May 10, 2020, from https://www.nhc.gov.cn/xcs/s3578/202002/ b01ffd9cabfa41c5835d2774f3d038bb.shtml.

6. Roy, D., Tripathy, S., Kar, S. K., Sharma, N., Verma, S. K., \& Kaushal, V. (2020). Study of knowledge, attitude, anxiety \& perceived mental healthcare need in Indian population during COVID-19 pandemic. Asian Journal of Psychiatry, 51, 102083. https://doi.org/10.1016/j.ajp.2020.102083.

7. Zhong, B. L., Luo, W., Li, H. M., et al. (2020). Knowledge, attitudes, and practices towards COVID-19 among Chinese residents during the rapid rise period of the COVID-19 outbreak: A quick online cross-sectional survey. International Journal of Biological Sciences, 16(10), 1745-1752. https://doi.org/10.7150/ijbs.45221.

8. Abdelhafiz, A. S., Mohammed, Z., Ibrahim, M. E., et al. (2020). Knowledge, perceptions, and attitude of Egyptians towards the novel coronavirus disease (COVID-19). Journal of Community Health. https://doi.org/10.1007/s10900-020-00827-7.

9. Rahul, R., \& Ranjan, G. K. (2020). Knowledge regarding prevention of novel coronavirus (COVID-19): An electronic crosssectional survey among Selected rural community. International Journal of Trend in Scientific Research and Development, 4(3), 422-425.

10. Haque, T., Hossain, K. M., \& Bhuiyan, M. M. R., et al. (2020). Knowledge, attitude and practices (KAP) towards COVID-19 and assessment of risks of infection by SARS-CoV-2 among the Bangladeshi population: An online cross-sectional survey. Retrieved May 10, 2020, from https://assets.researchsquare.com/ files/re-24562/v1/manuscript.pdf.

11. Wang, M. (2018). Investigation on the status of knowledge, attitude, practice of prevention and treatment of infectious diseases and the effect of health education intervention among residents. Chinese Rural Health Service Administration, 11(6), 960-962.

12. Wolf, M. S., Serper, M., Opsasnick, L., et al. (2020). Awareness, attitudes, and actions related to COVID-19 among adults with chronic conditions at the onset of the U.S. outbreak: A cross-sectional survey. Annals of Internal Medicine. https://doi. org/10.7326/M20-1239.

13. Zhang, M., Zhou, M., Tang, F., et al. (2020). Knowledge, attitude, and practice regarding COVID-19 among healthcare workers in Henan, China. The Journal of Hospital Infection, 105(2), 183-187. https://doi.org/10.1016/j.jhin.2020.04.012.

14. Qi, Y., Chen, L., Zhang, L., Yang, Y., Zhan, S., \& Fu, C. (2020). Public practice, attitude and knowledge of Novel CoronavirusInfected Pneumonia. Journal of Tropical Medicine, 20(02), 145-149.

15. Luo, L., Zeng, X., Liao, X., \& Yang, Y. (2020). Disease cognition, coping style and exercise behavior among the public during novel coronavirus epidemic: An online survey. Chinese Journal of Public Health. https://doi.org/10.11847/zgggws1128079.

16. Geldsetzer, P. (2020). Use of rapid online surveys to assess people's perceptions during infectious disease outbreaks: A crosssectional survey on COVID-19. Journal of Medical Internet Research, 22(4), e18790. https://doi.org/10.2196/18790.

17. Zheng, J., Zhang, Y., Xu, Y., Yang, L., Lin, C., \& Zhu, L. (2020). Health literacy and demand for health education on novel coronavirus pneumonia epidemic among community residents in Fujian province: An online survey during epidemic outbreak. Chinese Journal of Public Health, 36(2), 160-164. https://doi. org/10.11847/zgggws1128092.

Publisher's Note Springer Nature remains neutral with regard to jurisdictional claims in published maps and institutional affiliations. 